\title{
RENDIMIENTO Y CALIDAD DE HOJAS DE Sttevia rebaudiana Bert. BAJO LA OFERTA EDAFOLÓGICA Y DOS NIVELES DE RADIACION EN CINCO REGIONES DE COLOMBIA
}

\section{YIELD AND QUALITY OF LEAVES OF Stevia rebaudiana Bert. UNDER PEDOLOGICAL OFFER AND TWO LEVELS OF RADIATION IN FIVE COLOMBIAN REGIONS}

\author{
Jaime L. Hernández ${ }^{1}$, Enrique M. Combatt ${ }^{2}$, Alfredo Jarma O. ${ }^{3}$, Janer Polo S. ${ }^{4}$, Luis Rodríguez P.
}

${ }^{1}$ Ingeniero Agrónomo, M.Sc. Mz 8 lote 11, villa Sorrento, Montería, Córdoba, e-mail: jhernandez@fca.edu.co; ${ }^{2}$ Ingeniero Agrónomo, Ph.D. Universidad de Córdoba, carrera 6a No. 76-103, Montería, Córdoba-Colombia, e-mail: ecombatt@fca.edu. co, Autor para correspondencia; ${ }^{3}$ Ingeniero Agrónomo, M.Sc. Ph.D. Universidad de Córdoba, carrera 11 No. 62B-47, Montería, Córdoba-Colombia, e-mail: jarma@fca.edu.co; ${ }^{4}$ Ingeniero Agrónomo, M.Sc. Universidad de Córdoba, e-mail: jpolo@ fca.edu.co; ${ }^{5}$ Ingeniero Agrónomo, M.Sc. Universidad de Córdoba, Barrio P-5, transversal 12 No. 10-2, Montería, Córdoba, e-mail: larguez@fca.edu.co

Rev. U.D.C.A Act. \& Div. Cient. 19(1): 77-85, Enero-Junio, 2016

\section{RESUMEN}

El uso actual de estevia, a escala industrial, se ha incrementado a nivel mundial, argumento que conlleva a buscar zonas de mejor adaptación del cultivo, en muchas regiones del país. El objetivo de este trabajo fue analizar la estabilidad de los rendimientos y los contenidos de glucósidos de esteviol de Stevia rebaudiana Bert., en función de la oferta de luz de cinco regiones de Colombia. En cada sitio, se construyeron cinco parcelas experimentales del cultivar Morita II, en las localidades de Montería, Córdoba; Campamento, Antioquia; Palmira, Valle del Cauca; Fonseca, La Guajira y Valledupar, Cesar, bajo un diseño de bloques completos al azar, con dos tratamientos de radiación difusa y directa, con cinco repeticiones. Se cuantificaron los contenidos de esteviósido (Stv), rebaudiósido A (RebA), glucósidos principales (GP) en mg. $\mathrm{kg}^{-1}$ de hoja seca, por cromatografía líquida de alta eficacia; la relación RebA/Stv y el rendimiento de hoja seca, en kg.ha ${ }^{-1}$. Se realizó análisis de varianza y prueba de comparación de medias de Duncan $(\alpha=0,05)$. Los niveles de radiación no afectaron la síntesis de Stv y RebA, tampoco se presentó interacción entre los niveles de radiación de las localidades. Se registraron diferencias significativas en los contenidos de Stv, RebA y la relación RebA/Stv, entre las localidades. En Palmira, con $132,26 \mathrm{mg} \mathrm{kg}^{-1}$, se obtuvieron los mayores contenidos de RebA, mientras que la mayor relación RebA/Stv de 10:1 $\mathrm{mg} \mathrm{g}^{-1}$, que define la calidad de la hoja, se observó en Montería.
Palabras clave: Glucósidos de diterpeno, esteviósido, rebaudiósido A, interacción genotipo ambiente, radiación fotosintéticamente activa.

\section{SUMMARY}

The current use at industrial-scale of stevia has increased in the world, context that emphasizes the interest to search best areas for planting the crop in many regions of the country. The objective of this work was to analyze the yield stability and content of steviol glycosides of Stevia rebaudiana Bert. in function of light offer in five regions of Colombia. Five experimental plots of the cultivar Morita II were selected, located in Monteria-Cordoba; Campamento - Antioquia; Palmira - Valle del Cauca; Fonseca - Guajira and Valledupar - Cesar, under a complete random block design with two treatments, diffuse and direct radiation, with five replicates for each locality. The content of stevioside (Stv), rebaudioside A (RebA), main glycosides (GP) in mg. $\mathrm{kg}^{-1}$ of dry leaf, with a liquid chromatography of high-efficacy and the relationship $\mathrm{Reba} / \mathrm{Stv}$ and yield in $\mathrm{kg} \cdot \mathrm{ha}^{-1}$ of dry leaf was quantified. Data were analyzed through analysis of variance and Duncan's mean comparison test $(\alpha=0.05)$. The analysis of variance indicated that radiation levels did not affect the synthesis of Stv and RebA and no interaction between radiation levels by locality was detected. Significant differences in the content of Stv, RebA and relation RebA/Stv between the localities were determined. At Palmira, with $132.26 \mathrm{mg} \mathrm{kg}^{-1}$ the highest content of RebA was recorded, while the major relationship 
RebA/Stv, which defines the leaf quality, of $10: 1 \mathrm{mg}^{-g^{-1}}$ was obtained at Monteria.

Key words: Diterpene glycosides, stevioside, rebaudioside A, genotype environment interaction, photosynthetically active radiation.

\section{INTRODUCCIÓN}

La alta variabilidad de las condiciones ambientales en Colombia permite producir hoja de estevia durante todo el año; sin embargo, esta alta inestabilidad también genera zonas no aptas para su cultivo. En Colombia existen registros de cultivos en Antioquia, en Córdoba, en Tolima, en Huila, en Valle del Cauca y en Meta (Jarma, 2008), regiones que poseen diferencias considerables en la oferta edáfica y ambiental. La estevia posee gran capacidad de adaptación, ya que se puede cultivar desde 300 hasta 3000 msnm, según lo expuesto por Madan et al. (2010) y Yadav et al. (2011), con rangos de precipitación entre 1200 y $2000 \mathrm{~mm}$ anuales, niveles de temperatura entre 9 y $26^{\circ} \mathrm{C}$, de acuerdo con Singh \& Rao (2005) y humedad relativa de $85 \%$; a pesar de esto, es posible que la producción de los glucósidos de esteviol se vea afectada por las variaciones de las condiciones ambientales en los distintos lugares donde es cultivada (Jarma et al. 2005).

Los genotipos cultivados en Colombia se conocen popularmente como Morita 1 y Morita 2, de los cuales, poco se ha estudiado su estabilidad fenotípica; según Jarma et al. (2011), lo anterior implica un alto riesgo derivado de su variabilidad genética estrecha que, asociada a la alta variabilidad ambiental del país, los hace sensibles a factores, tanto bióticos como abióticos.

La estevia se está estableciendo en muchas partes del mundo, debido a sus propiedades terapéuticas y edulcurantes; es así como el glucósido de esteviol, que es una molécula de la estevia, se ha introducido en el mercado europeo como aditivo alimentario (Wolwer-Rieck, 2012). Serfaty et al. (2013) explican que el contenido de glucósidos de esteviol en estevia se determina en función de su concentración en las diferentes partes de la planta y la acumulación de biomasa; sin embargo, el desarrollo fenológico, la acumulación y la distribución de biomasa son notablemente afectadas por variaciones en las condiciones ambientales. Jaitak et al. (2008) encontraron que los edulcorantes de estevia registran valores entre 3,3 a 6,7\%, de esteviósidos y 1,2 a 2,3\%, de Rebaudiósido A.

Las variaciones en brillo solar, en temperatura, en radiación incidente y los factores edáficos, afectan el inicio de la floración en estevia que, a su vez, incide sobre los contenidos de glucósidos de esteviol, ya que en este punto se da la máxima acumulación de estas moléculas en las hojas, además de causar cambios en las expresiones fenotípicas y fisiológicas de la planta (Ceunen \& Geuns, 2013; Jarma, 2008; Ramesh et al. 2006).

En Colombia, se están estableciendo cultivos de estevia en diferentes lugares, sin que a la fecha se conozcan cuáles son las condiciones donde se presenta la mejor oferta de suelos y de ambientes para el establecimiento y obtener mayor rendimiento del cultivo. Esta investigación tuvo por objetivo cuantificar y analizar los contenidos de esteviósido, rebaudiósido A y los rendimientos de hoja de estevia, bajo dos niveles de radiación, en función de la oferta ambiental, de cinco regiones de Colombia.

\section{MATERIALES Y MÉTODOS}

Diseño experimental. Se seleccionaron cinco lotes comerciales correspondientes a cinco localidades; Palmira (Valle del Cauca), Campamento (Antioquia), Montería (Córdoba), Fonseca (La Guajira) y Valledupar (Cesar), a una altitud de 1001, 1700, 18, 12 y 168msnm, respectivamente. En cada una, se delimitaron parcelas experimentales, bajo la estructura de un diseño de bloques completos al azar, con dos tratamientos de radiación incidente (RI), T1 $=47 \%$ (Polisombra, conocida como polisombra al $50 \%$ ) y T2 $=100 \%$.

La tabla 1 muestra los niveles de radiación, evaluados en cada tratamiento y localidad, así como las condiciones de temperatura, de precipitación y de brillo solar, observadas durante el periodo de 6 meses, que se realizó la investigación. En estas localidades, se encontraron que el pH osciló entre 4,52, en Campamento, Antioquia y 7,58, en Fonseca, La Guajira y contenidos de fósforo, entre 125 y 5,9mg. $\mathrm{kg}^{-1}$, en estas mismas localidades. Los mayores contenidos de bases de intercambio, como Ca, K y Na $(20,12 ; 1,96$ y $0,92 \mathrm{cmolc}_{\mathrm{cg}}^{-1}$ ) se registraron en Fonseca y en La Guajira y los menores contendidos, en Campamento, Antioquia,

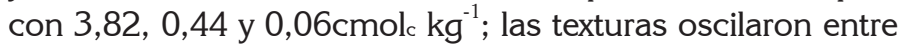
franco arenosas y arcillosas. Con base en la oferta ambiental de las cinco regiones estudiadas y para efectos de la discusión de resultados más claros, los ambientes se agruparon en condiciones de radiación y de temperaturas máximas y mínimas, que se pueden considerar como altas (Fonseca y Valledupar), intermedias (Montería y Palmira) y bajas (Campamento).

Cada experimento estuvo constituido por cinco unidades experimentales (UE), de $10 \mathrm{~m}^{2}$ cada una $\left(62,5\right.$ plantas $\left.\mathrm{m}^{-2}\right)$, para un total de 10 UE por localidad, que se establecieron como cinco repeticiones para cada nivel de radiación, por localidad. El genotipo utilizado fue Morita II.

En cada UE, se seleccionaron cinco plantas sanas en competencia y de similar edad fisiológica de crecimiento y se cuantificaron los contenidos de esteviósido (Stv), rebaudiósi- 
Tabla 1. Variables ambientales de las localidades de Colombia, donde se estableció el cultivo de estevia, Stevia rebaudiana Bertoni.

\begin{tabular}{|c|c|c|c|c|c|c|c|}
\hline \multirow{2}{*}{ Localidad } & RI $100 \%$ & RI $50 \%$ & Tmax & Tmin & Tprom & \multirow{2}{*}{$\frac{\mathbf{P}}{\mathrm{mm}}$} & \multirow{2}{*}{$\frac{\text { BS }}{\text { horas }}$} \\
\hline & \multicolumn{2}{|c|}{$\mu$ moles fotones. $\mathrm{m}^{-2} \cdot \mathrm{s}^{-1}$} & \multicolumn{3}{|c|}{-------------- ${ }^{\circ} \mathrm{C}-----------$} & & \\
\hline Palmira (Valle del Cauca) & 790 & 395 & 31,9 & 16,1 & 23,7 & 1065 & 1946 \\
\hline Montería (Córdoba) & 834 & 417 & 35,5 & 20,9 & 28 & 1247 & 2180 \\
\hline
\end{tabular}

RI: radiación incidente; Tmax: temperatura máxima; Tmin: temperatura mínima; Tprom: temperatura promedio; P: precipitación y BS: brillo solar anual.

do A (RebA) en $\mathrm{mg} \mathrm{g}^{-1}$ de peso seco de la hoja, por el método de cromatografía líquida de alta eficacia (HPLC), tal como lo muestra el cromatograma de la figura 1. Las condiciones de operación que se definieron y que fueron usadas para la calibración con los estándares puros y las muestras de las cinco regiones son las siguientes: Fase móvil: Acetonitrilo (ACN): Agua $\left(\mathrm{H}_{2} \mathrm{O}\right)$, 70:30; Isocrático; Flujo de trabajo: $500 \mu \mathrm{L} / \mathrm{min}$; Volumen de muestra inyectado: $5 \mu \mathrm{L}$; Detector PDA - 210nm; Tiempo de corrida: 20 min; Temperatura: ambiente. A partir de los resultados, se estimó la relación RebA/ Stv para cada localidad y se cuantificó el rendimiento de hoja seca por hectárea (REND) en el primer corte, por cada unidad experimental en gramos y convertido a kg.ha ${ }^{-1}$. La cosecha en cada localidad, se realizó en el momento antes de la floración, con la aparición de los primeros botones florales.

Análisis de datos. La información, se procesó por medio de análisis de varianza individual para cada localidad, bajo el modelo planteado; posteriormente, luego de verificar la homogeneidad de varianzas y el cumplimiento de supuestos, se implementó un análisis de varianza combinado entre localidades, para analizar la diferencia entre localidades (L), niveles de radiación (NR) y su interacción. Se realizó la prueba de comparación de medias de Duncan al 5\%, utilizando el paquete estadístico SAS (versión 9.1).

\section{RESULTADOS Y DISCUSIÓN}

Los contenidos de glucósidos principales (GP) oscilaron entre $10,26 \%$, para la localidad de Fonseca y $15,26 \%$, para Palmira, al 50\% de la radicación incidente. Entre los niveles de radiación no se presentaron diferencias $(12,99 \pm 0,06 \mathrm{mg}$ $\left.\mathrm{kg}^{-1}\right)$, aunque estas sí fueron evidentes entre localidades $(P \leq 0,05)$, siendo los ambientes denominados como intermedios; Palmira y Montería, los de mayor concentración de estas moléculas (Tabla 2).

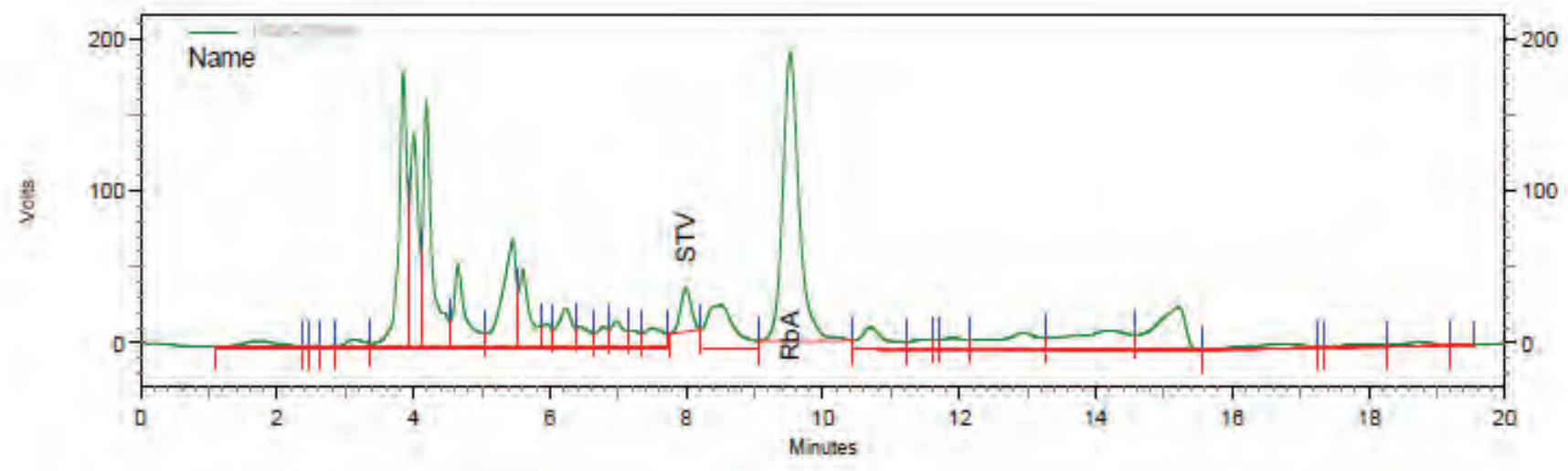

Figura 1. Cromatograma de muestra de hoja de estevia analizada. 
Tabla 2. Contenido de glucósidos principales en hojas de estevia, Stevia rebaudiana Bertoni, bajo dos condiciones de radiación incidente, en cinco ambientes de Colombia.

\begin{tabular}{|l|c|c|c|}
\hline \multicolumn{1}{|c|}{ Localidad } & $\mathbf{1 0 0 \%} \mathbf{R I}$ & $\mathbf{5 0 \%} \mathbf{R I}$ & Promedios de GP \\
\hline Palmira (Valle de Cauca) & $14,84 \mathrm{a}$ & $15,62 \mathrm{a}$ & $15,23 \mathrm{a}$ \\
\hline Montería (Córdoba) & $15,20 \mathrm{a}$ & $14,99 \mathrm{a}$ & $15,10 \mathrm{a}$ \\
\hline Campamento (Antioquia) & $12,65 \mathrm{~b}$ & $12,55 \mathrm{~b}$ & $12,60 \mathrm{~b}$ \\
\hline Valledupar (Cesar) & $10,50 \mathrm{~b}$ & $11,85 \mathrm{~b}$ & $11,18 \mathrm{~b}$ \\
\hline Fonseca (La Guajira) & $11,46 \mathrm{~b}$ & $10,26 \mathrm{~b}$ & $10,86 \mathrm{~b}$ \\
\hline
\end{tabular}

Promedios con la misma letra en una columna son iguales estadísticamente, de acuerdo al test protegido de Duncan (0,05\%).

Aunque el contenido de los GP es similar a lo reportado en otras partes del la relación entre las moléculas que componen los GP (RebA+Stv), varía significativamente, demostrando el efecto diferencial del ambiente, tal como lo afirman Jarma et al. (2005).

Los resultados del análisis de varianza combinado que compararon el contenido de estos dos glucósidos y los rendimientos de hoja seca por hectárea, se muestran en la tabla 3. Se puede observar que todas las variables fueron afectadas significativamente por los efectos simples de la localidad y que solo el rendimiento fue influenciado por la disminución de la radiación en un $50 \%$. Al analizar la interacción de las localidades por niveles de radiación, se observó que ninguna de estas fue significativa.

Por su parte, los coeficientes de variación de las variables no superaron el $17 \%$, indicando la precisión de los datos experimentales, para condiciones de campo.
Como se indicó anteriormente, los resultados observados en el contenido de los GP, indican que la radiación no tuvo un efecto importante sobre la concentración de estos; sin embargo, al considerar independientemente el efecto de las localidades, se pudo constatar que hubo diferencias entre localidades para la concentración de Stv, siendo Campamento, en Antioquia, la de mayor promedio de esta molécula, con el siguiente orden Antioquia > Valle del Cauca > Cesar, Córdoba y La Guajira (Tabla 4).

Si los contenidos de esteviósido encontrados en este estudio se expresan en términos de porcentaje, equivalen a concentraciones entre 1 y $4 \%$, lo que estaría por debajo de los registrados por Geuns (2000), quien plantea que los esteviósidos están presentes en un rango de alrededor de 4 al $20 \%$, en la materia seca de las hojas de la planta y, además, indica que estos contenidos dependen, principalmente, de las características del cultivar y de los factores ambientales; no obstante, es importante aclarar que esta reducción del Stv se hace

Tabla 3. Cuadrados medios para concentración de glucósidos (mg. $\mathrm{g}^{-1}$ de hoja) y rendimiento (kg.ha ${ }^{-1}$ ) en estevia, Stevia rebaudiana Bertoni, plantada en cinco localidades de Colombia, bajo dos niveles de radiación.

\begin{tabular}{|c|c|c|c|c|c|c|}
\hline FV & GL & GP & Stv & RebA & RebA/Stv & REND \\
\hline LOC & 4 & $387,88 * *$ & $962,72 * *$ & $4925,01 * *$ & $71,65 * *$ & $12236833,67 * *$ \\
\hline BLO (LOC) & 5 & $258,17^{\mathrm{ns}}$ & $3,81^{\mathrm{ns}}$ & $230,59^{\text {ns }}$ & $1,10^{\mathrm{ns}}$ & $201684,38^{\text {ns }}$ \\
\hline NR & 1 & $27,96^{\mathrm{ns}}$ & $7,64^{\mathrm{ns}}$ & $6,36^{\mathrm{ns}}$ & $1,91^{\mathrm{ns}}$ & $451350,27 * *$ \\
\hline LOC*NR & 4 & $214,19^{\mathrm{ns}}$ & $3,56^{\mathrm{ns}}$ & $198,36^{\mathrm{ns}}$ & $1,31^{\mathrm{ns}}$ & $126710,09^{\mathrm{ns}}$ \\
\hline ERROR & 29 & 184,85 & 6,53 & 138,73 & 0,92 & 92544,36 \\
\hline TOTAL & 43 & 535,99 & 94,91 & 597,12 & 7,58 & 1246459,36 \\
\hline MEDIA & & 129,99 & 20,11 & 109,87 & 6,54 & 1262,74 \\
\hline CV & & 10,45 & 12,71 & 10,71 & 14,73 & 17,03 \\
\hline
\end{tabular}

${ }^{\mathrm{ns}}=$ no significativo; *=significativo al 5\%; **= altamente significativo al 5\%; Stv: esteviósido; RebA rebaudiósido A; RebA/Stv: calidad de la hoja; LOC: localidades; NR: nivel de radiación. 
Tabla 4. Contenido porcentual de esteviósido (Stv), rebaudiósido A (RebA) y relación RebA/Stv en hojas de estevia, Stevia rebaudiana Bertoni, plantada en cinco ambientes de Colombia.

\begin{tabular}{|l|c|c|c|}
\hline \multicolumn{1}{|c|}{ Localidad } & RebA & Stv & RebA/Stv \\
\hline & ------ \\
\hline Palmira (Valle de Cauca) & $13,23 \mathrm{a}$ & $2,00 \mathrm{~b}$ & $6,6 \mathrm{~b}$ \\
\hline Montería (Córdoba) & $13,74 \mathrm{a}$ & $1,34 \mathrm{c}$ & $10,3 \mathrm{a}$ \\
\hline Campamento (Antioquia) & $8,66 \mathrm{~b}$ & $3,94 \mathrm{a}$ & $2,2 \mathrm{c}$ \\
\hline Valledupar (Cesar) & $9,99 \mathrm{~b}$ & $1,62 \mathrm{c}$ & $6,2 \mathrm{~b}$ \\
\hline Fonseca (La Guajira) & $9,46 \mathrm{~b}$ & $1,40 \mathrm{c}$ & $6,7 \mathrm{~b}$ \\
\hline
\end{tabular}

Promedios con la misma letra en una columna son iguales estadísticamente, de acuerdo al test protegido de Duncan (0,05\%).

a expensas de aumento en los contenidos porcentuales de RebA, como se discutirá más adelante.

Jarma et al. (2012) indican que aún se desconoce gran parte de cómo la planta traduce los estímulos de temperaturas en señales particulares; lo cierto es que las altas temperaturas inducen cambios y adaptaciones, a través de un regulador transcripcional (bHLH) denominado, frecuentemente, como factor interactuante del fitocromo 4 (PIF4), que está regulado por el genotipo e integra múltiples estímulos ambientales, durante el desarrollo de la planta (Koini et al. 2009).

Es posible que la variación de los contenidos de esteviósidos entre las localidades pueda estar explicada por las variaciones de la temperatura y el brillo solar entre las localidades, ya que estos dos factores influyen sobre la fotosíntesis, la respiración y muchos otros procesos metabólicos en la planta, incluida la síntesis de las principales moléculas edulcorantes (Jarma et al. 2012; Bondarev et al. 2003; Ceunen \& Geuns, 2013). Lo anterior podría explicar los resultados, porque en la localidad de Campamento se presentaron la temperatura y el brillo solar más bajos de las cinco localidades, con un promedio de $19,5^{\circ} \mathrm{C}$ y 1391 horas año ${ }^{-1}$, como se observa en la tabla 2, mientras que las localidades Montería, Valledupar y Fonseca, con mayor temperatura y brillo solar promedio, fueron precisamente las que registraron los menores contenidos de Stv en la hoja, notándose una relación inversa entre temperatura y brillo solar con los contenidos de Stv, tal vez porque la exposición prolongada de la planta a altas temperaturas y altos niveles de radiación, afecta negativamente la síntesis de la molécula.

El análisis de varianza combinado mostró diferencias altamente significativas, solamente para el contenido de RebA, entre localidades (Tabla 3). La prueba de comparación de medias señala que las localidades que presentaron mayor síntesis de RebA fueron Montería $\left(137,13 \mathrm{mg} \cdot \mathrm{g}^{-1}\right)$ y Palmira $\left(132,26 \mathrm{mg} \cdot \mathrm{g}^{-1}\right)$, seguidas de Valledupar $\left(95,71 \mathrm{mg} \cdot \mathrm{g}^{-1}\right)$, Fonseca $\left(94,44 \mathrm{mg} \cdot \mathrm{g}^{-1}\right)$ y Campamento $\left(86,56 \mathrm{mg} \mathrm{g}^{-1}\right)$, tal como lo registra la tabla 5 . El análisis de varianza combinado no mostró diferencias estadísticas entre los niveles de radiación del 50 y 100\%, indicando que las variaciones en estos niveles no afectaron la síntesis de rebaudiósido A; tampoco, se registró interacción de localidades por nivel de radiación, para esta variable.

Los resultados de esta investigación coinciden con trabajos adelantados por Jarma et al. (2012), quienes informaron de una relación inversa entre la concentración de RebA y altas temperaturas (máximas y mínimas) y altos niveles de radiación, como se presentan en Valledupar y Fonseca, de tal manera que valores altos de las tres variables ambientales inducirían valores bajos de RebA y que en los ambientes donde esta oferta ambiental es intermedia (Montería y Palmira), se favorece la síntesis de RebA; sin embargo, en Campamento, esta lógica no es evidente, debido, probablemente, a los altos niveles de sombreamiento ambiental que se presentan naturalmente, que reducirían, de manera importante, las tasas de fotosíntesis y, consecuentemente, bajos niveles de glucósidos totales, como se muestra en la tabla 2. Entonces, se podría considerar a Montería y a Palmira, como las localidades con mejor oferta ambiental, para la síntesis de RebA.

Estudios sobre la actividad del fitocromo en estevia, realizados por Ceunen et al. (2012), sugieren que las condiciones ambientales, sobretodo el fotoperiodo, la radiación y la temperatura influyen considerablemente en el desarrollo fenológico de la estevia, incluida la acumulación de las principales moléculas edulcorantes. Asimismo, la edad fisiológica juega un papel importante, ya que es, en el momento entre la formación de la yema y la iniciación de la floración, donde se da un gran incremento en los contenidos de los glucósidos (Vanidze et al. 2009) y esta etapa fenológica podría estar determinada por las unidades de calor acumuladas, las cua- 
les, están definidas como la diferencia entre la temperatura ambiente y la temperatura base de crecimiento de la planta (Ruiz et al. 2002).

Al respecto, Bondarev et al. (2003) advierten que se afectan procesos básicos del metabolismo de la planta, por lo que es posible que al ser RebA sintetizado por la ruta de los terpenos (Azcón-Bieto \& Talón, 2008), la susceptibilidad de esta vía a variaciones de luz (Jarma et al. 2012) y temperatura (Taiz \& Zeiger 2010), influyan de forma diferenciada en la síntesis de esta molécula, cuando es sometida a una oferta ambiental diversa; sin embargo, en contrastes con lo reportado por Jarma et al. (2012), en este estudio, no se encontraron diferencias para los niveles de radiación, por lo que, probablemente, la disimilitud entre los contenidos de RebA, para las localidades, pudieron estar dadas por otros factores ambientales, que afectan el desarrollo fenológico y el metabolismo secundario de la estevia o por la disponibilidad de nutrientes en cada localidad, ya que estos también afectan la síntesis y la acumulación de glucósidos de esteviol, como lo mencionan Bondarev et al. (2003).

El análisis de la relación RebA/Stv, en la tabla 3, indica que se presentaron diferencias estadísticas entre localidades, pero no entre niveles de radiación. La prueba de comparación de medias señaló que en Montería, se presentó la mayor relación RebA/Stv, con un promedio de 10,3:1, seguida de Fonseca, con 6,7:1, Palmira 6,6:1, Valledupar 6,2:1 y Campamento 2,2:1 (Tabla 4).

Estos resultados discrepan con los de Gardana et al. (2010), que referencian un contenido de Rebaudiósido A, en concentraciones de 2 a $4 \%$ y de esteviósido, de 4 al13\%. Asimismo, De Oliveira et al. (2007) exponen que la calidad de la hoja se determina por la relación RebA/Stv que, generalmente, está alrededor de 0,4 , obteniendo un producto de mejor calidad, en la medida que esta relación sea igual o mayor de 1 (De Oliveira et al. 2007; Carakostas et al. 2008). Cabe resaltar que en este estudio se encontró que en todas las localidades la relación fue superior a 1 .

La importancia de estimar la relación RebA/Stv, para este cultivo en Colombia, es que a nivel mundial se documentan concentraciones de RebA menores que las reportadas en esta investigación, por lo tanto, se puede deducir que en Colombia, se presentan condiciones edáfico ambientales, que favorecen el contenido de Rebaudiósido A en la hoja y, con ello, se logra un dulzor más agradable, contrario al sabor obtenido, cuando los contenidos de esteviósido son superiores, ya que este usualmente ofrece una sensación con características similares a un sabor amargo (Mitchell, 2006).

Por lo anterior, si se logra incrementar la siembra de este cultivo con rendimiento en hoja de estevia con estos atributos, se podría ofrecer a los productores una ventaja competitiva en el mercado de este producto.

De igual forma, las concentraciones de glucósidos principales (Stv + RebA), en todas las localidades, superan el promedio mundial, donde los edulcorantes de estevia registran valores entre 3,3 a 6,7\%, de esteviósidos y 1,2 a $2,3 \%$, de Rebaudiósido A (Jaitak et al. 2008), mientras que en este estudio, se encontraron valores de esteviósidos, entre 1,34 a 3,94\% y de Rebaudiósido A, entre 8,66 a 13,74\%. Lo anterior indica que, a pesar de que se observaron diferencias estadísticas entre las localidades evaluadas, la estevia presenta buena capacidad de adaptación a las condiciones ambientales en estas localidades, lo que en términos generales, se traduce en una mayor proporción de glucósidos totales en la hoja y en una mayor calidad comercial de este producto.

Respecto a los resultados en rendimiento de hoja seca por hectárea en el primer corte, entre todas las localidades, se encontró que para niveles de radiación del $100 \%$, se obtuvo un promedio de $1364 \mathrm{~kg} \cdot \mathrm{ha}^{-1}$, mientras que para la radiación al $50 \%$, se presentó un promedio en rendimiento de $1161,46 \mathrm{~kg} \cdot \mathrm{ha}^{-1}$, resultados que concuerdan con los encontrados por Jarma et al. (2005), donde se indica que la mayor acumulación de materia seca de hojas se obtuvo cuando Morita II fue expuesta a $100 \%$ de radiación incidente.

Ante la respuesta positiva del rendimiento a un mayor nivel de radiación, Raffo \& Iglesias (2004) indican que la radiación fotosintéticamente activa captada por una planta, determina la cantidad de materia seca producida y la calidad de la producción, influyendo sobre el crecimiento y la productividad. Es posible que este genotipo (Morita II) responda de una manera más eficiente a la fijación de $\mathrm{CO}_{2}$ por unidad de superficie fotosintética y al envío de fotoasimilados a la hoja, ya que según Taiz \& Zeiger (2010), la cantidad de luz y $\mathrm{CO}_{2}$ determinan la respuesta fotosintética de las hojas y que, en algunas situaciones, esta respuesta fotosintética puede ser limitada por un inadecuado suministro de luz, de tal forma, que el aumento en el flujo fotónico implica un aumento proporcional de la tasa fotosintética.

Al realizarse la prueba de comparación de medias, se encontraron diferencias estadísticas entre los rendimientos obtenidos en cada localidad, siendo Palmira, donde se lograron los mayores, con $3477 \mathrm{~kg}$, de hoja seca por hectárea, en el primer corte de hoja; entre las demás localidades no se registraron diferencias estadísticamente significativas (Figura 2).

Estos resultados son comparables con los encontrados por Kandus et al. (2010), en maíz y por Rodríguez et al. (2011), en trigo, donde se indica que los genotipos tienen un comportamiento desigual en cada ambiente, donde se realizaron las investigaciones. Los distintos rendimientos entre las lo- 


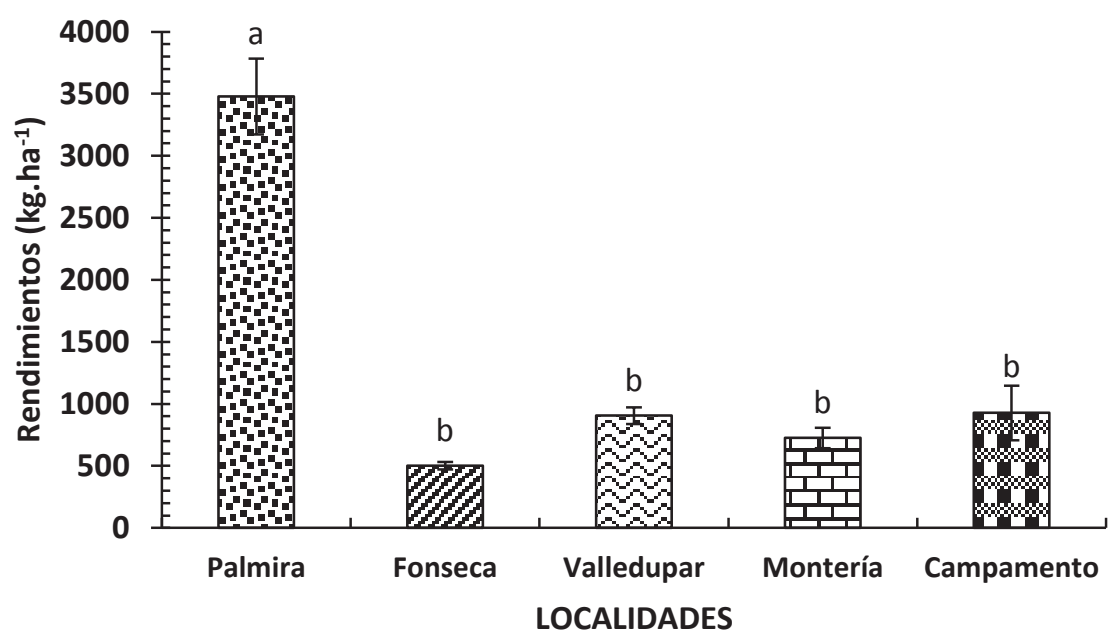

Figura 2. Rendimientos de hoja de estevia, Stevia rebaudiana Bertoni, plantada en cinco localidades de Colombia.

calidades que se determinaron, se pueden entender a partir de las diferentes condiciones ambientales, propias de cada localidad. Montemayor et al. (2006) y Serfaty et al. (2013) explican que diversas condiciones ambientales afectan notablemente el desarrollo y la acumulación de biomasa de plantas de estevia, debido a que los factores ambientales, como radiación solar, precipitación y temperatura, afectan las expresiones fenotípicas y fisiológicas de las plantas, como la fotosíntesis, la respiración, la división celular y el crecimiento celular, entre otras. Fuera de Mizukami et al. (1983), Ceunen \& Geuns (2013) indican que la irradiación, la temperatura y los nutrientes disponibles, también pueden afectar la acumulación de los glucósidos de steviol, en hojas de estevia.

De la presente investigación se puede concluir, que la disminución de radiación en un $50 \%$, no afectó la síntesis de los glucósidos en ninguna de las localidades, consideradas en el estudio.

La oferta edafoambiental de las diferentes localidades afectó diferencialmente la síntesis de esteviosido y rebaudiosido A, siendo Palmira y Montería las localidades con mayores valores, respecto a Campamento, Valledupar y Fonseca.

En Campamento, se presentó el mayor promedio de los contenidos de esteviósido, resultado que pudo ser influenciado por las bajas temperaturas y el bajo brillo solar de esta localidad.

En Montería y en Palmira, se registraron los mayores contenidos de RebA, debido, probablemente, por el efecto de las temperaturas y el brillo solar (promedio entre máximas y mínimas evaluadas).
Aunque la mejor calidad de hoja se obtuvo en Montería, donde existe mayor relación RebA/Stv, con 10,177:1 $\mathrm{mg}^{-1} \mathrm{~g}^{-1}$, los rendimientos de hoja seca, fueron mayores en Palmira.

Agradecimientos: Al Centro de Investigación CIUC de la Universidad de Córdoba y al Ministerio de Agricultura y Desarrollo Rural -CENIRED-, por la financiación del macro proyecto "Determinación de zonas agroecológicas óptimas en función del contenido de los glucósidos en Stevia rebaudiana en Colombia". Conflicto de interés: El manuscrito fue preparado y revisado con la participación de todos los autores, quienes declaramos que no existe conflicto de interés que ponga en riesgo la validez de los resultados presentados.

\section{BIBLIOGRAFÍA}

1. AZCÓN-BIETO, J.; TALÓN, M. 2008. Fundamentos de fisiología vegetal. McGraw-Hill Interamericana de España, Madrid. 325p.

2. BONDAREV, N.I.; SUKHANOVA, M.A.; RESHETNYAK, O.V.; NOSOV, A.M. 2003. Stevial Glycoside content in different organs of Stevia rebaudiana and it's dynamics during ontogeny. Biologia Plantarum. 47(2):261-264.

3. CARAKOSTAS, M.C.; CURRY, L.L.; BOILEAU, A.C.; BROSICK, D.J. 2008. Overwiew; the history, technical ocurring steviol glycoside, for use, in food and beverages. Food Chem. Toxicol. 7(3):SI-S10.

4. CEUNEN, S.; WERBROUCK, S.; GEUNS, J.M.C. 2012. Stimulation of steviol glycoside accumulation in Ste- 
via rebaudiana by red LED light. J. Plant Physiol. 169:749-752.

5. CEUNEN, S.; GEUNS, J.M.C. 2013. Influence of photoperiodism on the spatio-temporal accumulation of steviol glycosides in Stevia rebaudiana (Bertoni). Plant Science. 198(2):72-82.

6. CHATSUDTHIPONG, V.; MUANPRASAT, C. 2009. Stevioside and related compounds: therapeutic benefits beyond sweetness. Pharmacol. Therapeutics. 121:41-54.

7. DE OLIVEIRA, B.H.; PACKER, J.F.; CHIMELLI, M.; DE JESÚS, D.A. 2007. Enzymatic modification of stevioside by cell-free extract of Gibberella fujikuroi. J. Biotechnol. 131(1):92-99.

8. GARDANA, C.; SCAGLIANTI, M.; SIMONETTI, P. 2010. Evaluation of steviol and its glycosides in Stevia rebaudiana leaves and comercial sweetener by ultra-highperformance liquid chromatography-mass spectrometry. J. Chromatography A 1217(9):14631470.

9. GEUNS, J.M.C. 2000. Safety of stevia and stevioside. Recent Res. Developments. Phytochem. 4(2):75-88.

10. JAITAK, V.; GUPTA, A.; KAUL, A.V.; AHUJA, P. 2008. Validated high-performance thin-layer chromatography method for steviol glycosides in Stevia rebaudiana. J. Pharmac. Biomed. Analysis. 47(4-5):790-794.

11. JARMA, A.; CARDONA, C.; FERNÁNDEZ, C. 2012. Efecto de la temperatura y radiación en la producción de glucósidos de esteviol en Stevia rebaudiana en el Caribe húmedo colombiano. Rev. U.D.C.A Act. \& Div. Cient. 15(2):339-347.

12. JARMA, A.; ARAMENDIZ, H.; CLEVES, J.A. 2011. Estabilidad fenotípica y densidades de planta de genotipos de estevia (Stevia rebaudiana Bert.) en la región Caribe de Colombia. Acta Agronómica. 60 (2): 176182.

13. JARMA, A. 2008. Estudios de adaptación y manejo integrado de estevia (Stevia rebaudiana Bert.): nueva alternativa agroindustrial del Caribe colombiano. Una revisión. Rev. Col. Cienc. Hort. 2(1):109-120.

14. JARMA, A.; RENGIFO, T.; ARAMENDIZ, T.H. 2005. Aspectos fisiológicos de estevia (Stevia rebaudiana Bertoni) en el Caribe colombiano: I. Efecto de la ra- diación incidente sobre el área foliar y la distribución de biomasa. Agr. Col. 23(2):207-216.

15. KANDUS, M.; ALMORZA, D.; BOGGIO, R.; RONCEROS, D.; SALERNO, J.C. 2010. Statistical models for evaluating the genotype-environment interaction in maize (Zea mays L.). Phyton (B. Aires). 79(1):39-46.

16. KOINI, M.A.; ALVEY, L.; ALLEN, T.; TILLEY, C.A.; HARBERD, N.P.; WHITELAM, G.C.; FRANKLIN, K.A. 2009. High temperature-mediated adaptations in plant architecture require the bHLH transcription factor PIF4. Current Biology. 19(5):408-413.

17. MADAN, S.; AHMAD, S.; SINGH, G.N.; KOHLI, K.; KUMAR, Y.; SINGH, R.; GARG, M. 2010. Stevia rebaudiana (Bert.) Bertoni-a review. Ind. J. Nat. Prod. Resource. 1(1):267-286.

18. MITCHELL, H. 2006. Bulking Agents: Multi-functional Ingredients. In: Mitchell, H. (ed). Sweeteners and Sugar Alternatives in Food Technology. Blackwell Publ. $1^{\text {st }}$ ed. p.65-85.

19. MIZUKAMI, H.; SHIBA, K.; OHASHI, H. 1983. Effect of temperature on growth and stevioside formation of Stevia rebaudiana. Shoyakugaku Zasshi. 37:175179.

20. MONTEMAYOR, J.; ZERMEÑO, J.A.; OLAGUE, J.; ALDACO, R.; FORTIS, M.; SALAZAR, E.; CRUZ, J.; VÁSQUEZ, C. 2006. Efecto de la densidad y estructura del dosel de maíz en la penetración de la radiación solar. Rev. Int. Bot. Exp. (YTON). 75:47-53.

21. RAFFO, M.; IGLESIAS, D. 2004. Efecto de la intercepción y distribución de la radiación fotosintéticamente activa en manzanos cv. 'Fuji', bajo cuatro sistemas de conducción en alta densidad. Rev. Invest. Agropec. 33(2):29-42.

22. RAMESH, K.; VIRENDRA, S.; MEGEJI, N.W. 2006. Cultivation of Stevia rebaudiana (Bert.) Bertoni: a comprehensive review. Adv. Agron. 89(1):137-177.

23. RODRÍGUEZ, R.E.; PONCE, J.T.; RUEDA, E.O.; AVENDAÑO, L.; PAZ, J.J.; SANTILLANO, J.; CRUZ, M. 2011. Interacción genotipo-ambiente para la estabilidad de rendimiento en trigo en la región de Mexicali, B.C. México. Trop. Subtrop. Agroecosyst. 14:543-558.

24. RUIZ C., J.A.; FLORES L., H.E.; RAMÍREZ, D., J.L.; GONZALEZ E., D.R. 2002. Temperaturas cardinales 
y duración del ciclo de madurez del híbrido de maíz $\mathrm{H}-311$ en condiciones de temporal. Agrociencia. 36(5):569-577.

25. SERFATY, M.; IBDAH, M.; FISCHER, R.; CHAIMOVITSH, D.; SARANGA, Y.; DUDAI, N. 2013. Dynamics of yield components and stevioside production in Stevia rebaudiana grown under different planting times, plant stands and harvest regime. Industr. Crops Products. 50(2):731-736.

26. SINGH, S.; RAO, G. 2005. Stevia: The herbal sugar of 21st century. Sugar Technol. 71(2):17-24.

27. TAIZ, L.; ZEIGER, E. 2010. Plant physiology. $5^{\text {th }}$ ed. Sinauer Associates, Inc. Redwood City, CA. p.644-645.
28. VANIDZE, M.R.; KALANDIYA, A.G.; CHANUKVADZE, K.R. 2009. Identification and quantitative determination of the content of diterpene glycosides in stevia (Stevia rebaudiana Bertoni). Khim. Rastit. Syr'ya. 4(2):155-158.

29. YADAV, A.K.; SINGH, S.; DHYANI, D.; AHUJA, P.S. 2011. A review on the improvement of stevia [Stevia rebaudiana (Bertoni)]. Can. J. Plant Science. 91(3):1-27.

30. WOLWER-RIECK, U. 2012. The leaves of Stevia rebaudiana (Bertoni), their constituents and the analyses thereof: a review. J. Agr. Food Chem. 60:886-895.

Recibido: Agosto 20 de 2015

Aceptado: Marzo 19 de 2016

\section{Cómo citar:}

Hernández, J.L.; Combatt, E.M.; Jarma O., A.; Polo S., J.; Rodríguez P., L. 2016. Rendimiento y calidad de hojas de Stevia rebaudiana Bert. bajo la oferta edafológica y dos niveles de radiación en cinco regiones de Colombia. Rev. U.D.C.A Act. \& Div. Cient. 19(1): 77-85. 